\title{
The Control System of 100kv High Voltage Power Supply
}

\author{
Shengmin Pan ${ }^{a}$, Peng Fu ${ }^{b}$, Yiyun Huang ${ }^{c}$, Baocan He ${ }^{d}$, Hulin Feng ${ }^{e}$, \\ Denghui Wang ${ }^{f}$ \\ Institute of Plasma Physics Chinese Academy of Science He Fei, China \\ a psm@ipp.ac.cn, ${ }^{\mathrm{b}}$ fupeng@ipp.ac.cn, c $y$ yhuang@ipp.ac.cn, ${ }^{\mathrm{d}}$ hebaocan@ipp.ac.cn, \\ e hlfeng@ipp.ac.cn, ${ }^{f}$ wdenghui@ipp.ac.cn
}

Keywords: High voltage, control, monitor, fiber optic.

\begin{abstract}
It described a control system of $100 \mathrm{kV}$ high voltage power supply. It used PC control and monitor the power modules via fiber optic. By testing, proved the control system is a good isolation and provides stability control system. By this way, we can remote control the power modules.
\end{abstract}

\section{Introduction}

The $100 \mathrm{kV}$ high voltage power supply(HVPS) is developed for the national great science engineering superconducting tokamak fusion experimental device(EAST), which is a set of high power high voltage pulse power supply. The HVPS adopted Pulse Step Modulation technology[1], which connected by 144 the same structure of 770V/70A power supply module in series (Fig. 1). Its rated output is $100 \mathrm{kV} / 70 \mathrm{~A}$, maximum pulse width is $1000 \mathrm{~s}$. Due to the power supply's highest output voltage up to $120 \mathrm{kV}$, therefore, between the power supply module and the control system must through the reliable isolation to ensure the safety of the controllers. And because of it worked in pulse module, there are strong electromagnetic interference when the load lighter. Based on the above consideration, this control system used optical fiber as transmission medium[2][3], which is widely used in modern communication networks, and with strong anti-interference ability and the resistance to high voltage. Through the optical fiber communication between control PC and power supply module, to control and monitor the power supply module was realized.

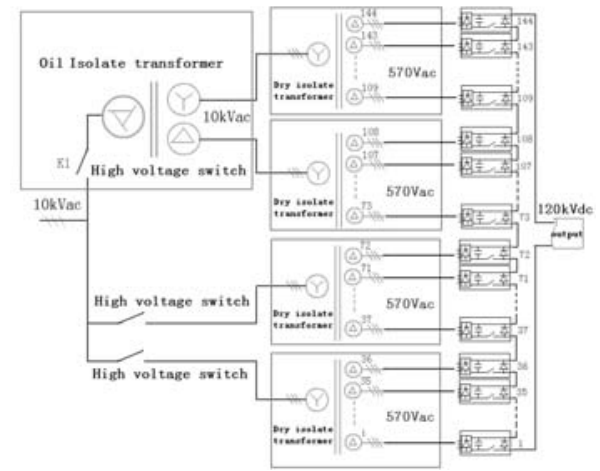

Fig.1 High voltage power supply topology

\section{The System Structure and Overview}

The control system includes the RS232 optical fiber network, 144 power supply module, control PC and monitor PC. Control system structure as shown in fig.2.

Each power module has RS232 communication port, internal intelligent controller(MCU) real-time acquisition internal condition and parameters of the power supply module. Through the optical fiber communication with monitoring PC, which read and write data by force control 6.0 software[4][5]. The monitoring PC can real-time monitoring the state of power supply module, and recording operation parameters and fault record of each power supply module by the database.

Each power module has an optical receiver, which connects with the photoelectric conversion box by optical fiber, through the optical signal to control power module on or off. Photoelectric 
conversion box connect with control PC by SCSI-100 cable. Control PC through the DO card to control the power module. The control PC adopts the output voltage of power supply by AD card at the same time.

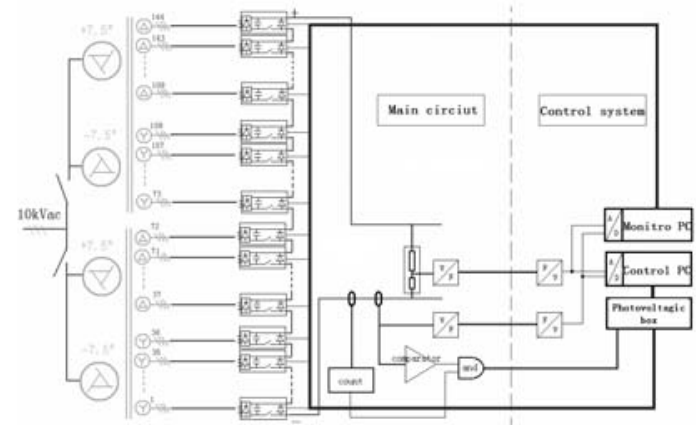

Fig.2 The control system structure diagram

\section{The Power Supply Module Internal Intelligent Controller}

Control system has local control and remote control mode[6]. Local control mode is mainly used for the operation of the single power module debugging. In this mode, we debug the single power module by buttons and adjustable potentiometer to set all kinds of reference. In remote control mode, the operator on the remote control room to control and monitor the power supply by software on control PC and monitor PC. The principle diagram of the internal intelligent controller is shown in fig.3.

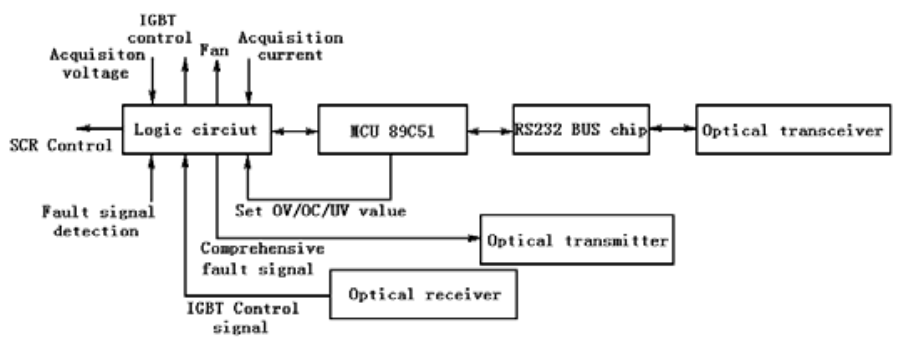

Fig.3 The principle diagram of the power supply module internal intelligent controller

The core control chip of the internal intelligent controller is single chip microcomputer 89C51[7][8], which control the power module IGBT, fan and vacuum contactor by logic control circuit, while detecting the power module output voltage, current and the fault signal.

In the local control mode, the operator through the adjustable potentiometer and key to set the power module over-current, over voltage and under-voltage values and parameters [9] related to the power module. At the same time, all kinds of faults signal can display by the light emitting diode.

In the remote control mode, the microprocessor is responsible for processing the command from control PC through RS232 communication. At the same time, control the power module according the control PC's command [10] [11] [12], and send the current and voltage value of the power supply and fault information to monitor PC. In this mode, the controller does not respond to local control mode, but the LED display all kinds of signal like the local control mode.

\section{RS232 BUS and Optical Fiber HUB}

There are multiple nodes in RS232 bus [13], specific number can be depending on the system needs to be. There are 144 nodes in the control system. Because the RS232 bus established a loop between the main circuit of the power supply and remote-control PC, there may lead to high voltage into the control system in abnormal state. In order to avoid this kind of circumstance happening, it used 50 meters optical fiber to connect control system and power supply module. So, the optical medium RS232 bus is a king of relatively perfect solution was used to realize communication between high voltage power supply and control system. 
The monitor PC must through RS232 conversion equipment to achieve communication with power module. This control system uses a serial port server from ADVANTECH for EKI-2816. EKI-2816 is a special serial port server [15], it can communicate between the monitor PC and power module through network port.

Optical HUB is used to connect with the optical transceiver module in the power module by optical fiber and change the bus signal to connect with EKI-2816 at the same time.

\section{IGBT Control Signal and the Module Fault Signal}

Control PC through the DO acquisition card (model CPCI-7248) to connect with the optical signal control box, then control the each power module by optical signals. Each power module controller has a fiber optic receiver, which used to receive IGBT control signal sent by control PC. The control signal directly into the logic control circuit to control the IGBT.

In order to control the output of the power supply voltage, designed a voltage feedback control box, which is used to compare the power supply output voltage and set voltage, then achieve the purpose of the output voltage.

Because of the high voltage power supply work in pulse condition, there will have very strong electromagnetic interference [16]. In order to avoid the MCU failure under the electromagnetic interference, there is a signal from all kinds of fault signal logic operations, which will send to monitor PC by the optical transmitter.

\section{The Experiment}

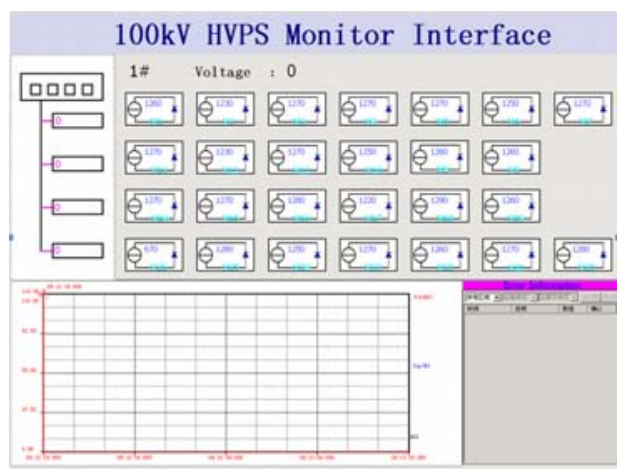

Fig.4 monitor interface

Experiments adopt 144 power modules in series, the control PC control the power supply module, the monitor PC monitor the power modules statement in real-time. Monitoring interface as shown in fig.4, it can real-time monitoring 144 power module, and the follow inspection time is less than 100 milliseconds.

Figure5(a) is the waveform for the power module switch on at the same time, waveform 1(yellow) is the voltage waveform $(1 \mathrm{~V}=20 \mathrm{kV}$ ), waveform 2 (green) is the set voltage value, waveform 3 (pruple) is the current waveform $(1 \mathrm{~V}=40 \mathrm{~A})$. From the waveform 1 , the highest output voltage is $80 \mathrm{kV}$, the rise time is less than 100us, and no overshoot on the rise along. From the waveform 3, the maximum current is $60 \mathrm{~A}$.

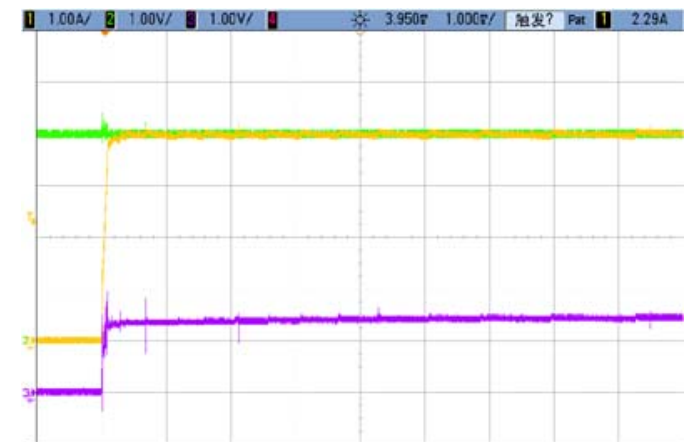

Fig. $580 \mathrm{kV}$ output and current waveform 


\section{Conclusion}

The control system through RS232 bus communication monitors each power supply module by monitor computer. Control PC through DO acquisition card communications with the power supply module, so power supply module can achieve remote control of the high voltage power supply. In order to ensure safety and increase the anti-interference ability, RS232 bus and control PC and power supply module panel communication transmission medium by the optical fiber. Operator by operating computer can realize the control and monitoring the power module. Based on the 144 series power module debugging, we can see that using optical fiber RS232 bus and distributed control network, fully meet the design requirements. And use this kind of control mode, the rise time of the power supply is less than 100us, and there is no over shoot.

\section{References}

[1]. U.E.Schwarz.Gigitized high power modulation[C], 14th Symposium on Fusion Technology, Avignon, 1986.

[2]. WANG Fu-sheng, LIU Xiao-ning, PAN Sheng-min, 3-phase current-sour-fectifier based on DSP and CPLD [J], Advanced Technology of Electrical Engineering and Energy, 2005-04, page: 33-39.

[3]. Ji Baojie, Zhen Caihong etc. Distributed Measurement and Control System Used in Agricultural Greenhouse Based on Single-chip controller [J], Computer Measurement and control, 2007-1, page: $73-76$.

[4]. Renso Wolf, HuWei, Stefan Bruins, Long Fengli, Ronnie Groen. Digital Power Supply Controller for control of extremely precise power supplies[J], Power Electronic and Applications,2007 European conference on 2-5 Sept.2007 page:1-6.

[5]. K.Strunz, P.Martinole, S.Muller, O.Huet. CONTROL SYSTEM TESTING IN ELECTRICITY MARKET PLACES[J], Power Industry Computer Applications, 2001. PICA 2001. Innovative Computing for Power-Electric Energy Meets the Market. 22nd IEEE Power Engineering Society International Conference on 20-24 May 2001. page:99-103.

[6]. M.O’Sullivan, N.Dobeck, R.Flood, J.Perry. CEBAF Beam Transport Magnet Power Supply Control System[J], Nuclear Science Symposium and Medical Imaging Conference ,1991, Conference Record of the 1991 IEEE 2-9 NOV. 1991.page:952-956 vol.2.

[7]. Zhang Chong, Li Aihua etc. Design of Intelligent Gun Cabinet Based on C8051F040 MCU [J], Computer Measurement and Control, 2012-11, page: 3048-3050. 